\title{
Canadian Urological Association guideline on androgen deprivation therapy: Adverse events and management strategies - Executive summary
}

\author{
Andrea Kokorovic, MD'; Alan I. So, MD2; Hosam Serag, MD²; Christopher French, MD; Robert J. Hamilton, MD"; \\ Jason P. Izard, MD'; Jasmir G. Nayak, MD'; Fréderic Pouliot, MD; Fred Saad, MD'; Bobby Shayegan, MD; \\ Armen Aprikian, $M D^{9}$; Ricardo A. Rendon, $M D^{10}$
}

\begin{abstract}
'Centre Hospitalier de I'Université de Montréal, Montreal, QC, Canada; ${ }^{2}$ Department of Urological Sciences, University of British Columbia, Vancouver, BC, Canada; ${ }^{3}$ Department of Surgery, Division of Urology, Memorial University, St. John's, NL, Canada; 'Division of Urology, Department of Surgery, Princess Margaret Cancer Centre, Toronto, ON, Canada; ' 5 Department of Urology, Queen's University, Kingston, ON, Canada; 'S Section of Urology, Department of Surgery, University of Manitoba, Winnipeg, MB, Canada; ${ }^{7}$ CHU de Quebec, Université Laval, Quebec City, QC, Canada; ${ }^{8}$ Department of Surgery (Urology) and Oncology, McMaster University, Hamilton, ON, Canada; ' $M$ McGill University Health Centre, Montreal, QC, Canada; ${ }^{10}$ Department of Urology, Dalhousie, University, Halifax, NS, Canada
\end{abstract}

Cite as: Kokorovic A, So Al, Serag H, et al. Canadian Urological Association Guideline on androgen deprivation therapy: Adverse events and management strategies - Executive Summary. Can Urol Assoc J 2021;15(6):159-61. http://dx.doi.org/10.5489/cuaj.7357

View full guideline at cuaj.ca and cua.org

A ndrogen deprivation therapy (ADT) plays an important role in the contemporary management of prostate cancer (PCa) across various stages of the disease. While ADT remains a highly effective treatment for $\mathrm{PCa}$, it is not curative, and its use is associated with significant adverse events that span across various organ systems.

\section{Cardiometabolic health}

\section{Summary of evidence}

- Cardiac complications:

- ADT may increase the risk of cardiac complications, especially in patients with preexisting cardiovascular disease (CVD) or a history of major adverse cardiac events (MACE).

- Thromboembolic and cerebrovascular events:

- ADT may increase the risk of venous thromboembolism (VTE) and stroke.

- Body composition:

- ADT is associated with changes in body composition, including increased body weight and fat mass, decreased lean body mass, and decreased muscle mass.

- Metabolic parameters:

- The metabolic complications of ADT include insulin resistance, glucose intolerance, and changes in lipid profile.
- ADT is associated with increased risk of incident diabetes and may worsen glycemic control in men with a pre-existing diagnosis.

- Men receiving ADT may be at risk for developing metabolic syndrome.

\section{Recommendations}

- The patient's primary care provider should be informed that the patient has been initiated on ADT, and that there may be adverse events associated with this therapy (Expert opinion).

- Lifestyle modifications (smoking cessation, dietary modifications, exercise) should be strongly encouraged (Expert opinion).

- Providers should obtain a comprehensive baseline physical examination prior to ADT initiation that includes blood pressure, weight, waist circumference, and calculation of body mass index (BMI) (Expert opinion).

- Providers should order baseline laboratory investigations, including fasting plasma glucose and lipid profile (triglycerides, low-density lipoprotein [LDL] cholesterol, high-density lipoprotein [HDL] cholesterol, and total cholesterol) (Expert opinion).

- Patients should be screened for diabetes with fasting plasma glucose, oral glucose tolerance test or hemoglobin (Hgb) A1c level (Expert opinion).

- Patients should have their blood pressure monitored and hypertension should be treated (Expert opinion).

- Dyslipidemia should be treated according to current best practice guidelines (Expert opinion).

- The above metabolic assessments should be continued at 6-12-month intervals throughout treatment duration (Expert opinion). 
- Patients should be encouraged to attend supervised exercise programs using a combination of resistance and aerobic training (Level of evidence [LE] 2, strong recommendation).

- In patients with a history of myocardial infarction or stroke, referral to a cardiologist or cardio-oncologist may be considered for assessment and medical optimization prior to initiating ADT (Expert opinion).

- Use of a gonadotropin-releasing hormone (GnRH) antagonist may be considered in men with a prior history of myocardial infarction or stroke (LE 2, weak recommendation).

\section{Bone health}

\section{Summary of evidence}

Use of ADT in men with PCa has detrimental effects on bone health, including decreased bone mineral density (BMD), osteoporosis, and increased risk for clinical fractures.

\section{Recommendations}

- A comprehensive history and physical examination to include fall risk and height measurement should be performed prior to initiating ADT (Expert opinion).

- Patients should be counselled regarding smoking and alcohol cessation (Expert opinion).

- Patients should be encouraged to participate in exercise therapy using a combination of resistance and aerobic training, preferably in a supervised setting ( $L E 2$, strong recommendation).

- Providers should obtain baseline calcium and 25-hydroxyvitamin D levels at the start of ADT (Expert opinion).

- Men should maintain adequate calcium intake (1200 mg PO daily from dietary sources and supplements) (Expert opinion).

- Vitamin D supplementation (800-2000 IU PO daily) should be initiated at the start of ADT (Expert opinion). Providers should screen men initiating long-term ADT for osteoporosis using BMD testing with dual energy x-ray absorptiometry (DXA) (as per the 2010 clinical practice guidelines for the diagnosis and management of osteoporosis in Canada) (Expert opinion).

- A 10-year major osteoporotic fracture risk using a validated tool should be calculated (Expert opinion).

- Men diagnosed with osteoporosis, those with history of fragility fractures in the hip or spine, those with a history of multiple fragility fractures, or those with a moderate or high 10-year fracture risk should be treated with a bisphosphonate or denosumab at doses recommended for the general population ( $L E 1$, strong recommendation).

- DXA should be repeated every 2-3 years in men at low risk for fractures receiving ADT. In men with osteopenia or those at moderate or high risk for fractures, DXA should be repeated every 1-2 years until treatment cessation. Patients started on pharmacological therapy should have followup DXA to assess for treatment response (Expert opinion).

\section{Hot flashes}

Summary of evidence

Hot flashes are a common and bothersome side effect of ADT.

\section{Recommendations}

- Patients should be counselled on identification and avoidance of potential triggers (Expert opinion).

- The best pharmacological therapy to treat hot flashes remains unclear, however, several agents have shown to be effective and may be considered for use ( $L E 2$, weak recommendation).

- Use of intermittent ADT improves hot flashes and should be considered in appropriately selected patients (LE 2, strong recommendation).

- Acupuncture may have a beneficial effect and can be considered in patients unwilling or unable to use pharmacotherapy ( $L E 3$, weak recommendation).

\section{Breast events}

\section{Summary of evidence}

ADT-related breast events include gynecomastia and mastodynia. Gynecomastia occurs most commonly with antiandrogen (AA) monotherapy and is a rare complication of luteinizing hormone-releasing hormone (LHRH) monotherapy or combined androgen blockade.

\section{Recommendations}

- Prophylaxis for the prevention of gynecomastia in men receiving $\mathrm{ADT}$ is not currently recommended (Expert opinion).

- Tamoxifen or radiation therapy (RT) may be used for prevention and treatment of breast events in men receiving bicalutamide monotherapy; tamoxifen is more effective than $\mathbf{R T}$ ( $L E 1$, strong recommendation). 


\section{Cognitive function}

\section{Summary of evidence}

Use of ADT in men with PCa may be associated with changes in cognition, depression, and development of dementia; however, evidence related to causality remains weak and further prospective data are needed.

\section{Recommendations}

- Men receiving ADT should be monitored for cognitive decline and depression throughout duration of treatment (Expert opinion).

\section{Fatigue and anemia}

\section{Summary of evidence}

Fatigue is a noticeable side effect of ADT and the underlying cause is often multifactorial. Anemia occurs commonly in men receiving ADT but is mild in most cases and often does not warrant treatment.

\section{Recommendations}

- Men experiencing fatigue should be counselled to participate in exercise therapy ( $L E 2$, strong recommendation).

- Men with severe anemia or those with a decline in hemoglobin that exceeds the expected response to ADT alone should be referred for further evaluation (Expert opinion).

\section{Sexual function}

\section{Summary of evidence}

ADT impacts multiple domains of sexual function, including body image, loss of libido, and erectile function.

\section{Recommendations}

- In men desiring improved sexual function, referral to a sex therapist for multimodal treatment should be considered (Expert opinion).

- Intermittent ADT may improve libido and erectile function and should be considered in appropriately selected patients (LE 1, strong recommendation).

\section{Health-related quality of life}

\section{Summary of evidence}

Patients on ADT experience significant decrements in multiple health-related quality of life (HRQOL) domains.

\section{Recommendations}

- Exercise therapy should be encouraged in all men to improve $\mathrm{HRQOL}$ during treatment ( $L E 2$, strong recommendation).

- Intermittent ADT improves HRQOL and should be considered in appropriately selected patients ( $L E 1$, strong recommendation).

\section{Summary}

Patients initiating ADT require appropriate counselling regarding adverse effects. A partnership between the urologist and primary care providers using a multidisciplinary approach is imperative to mitigate complications that may occur in response to ADT. This has become increasingly important in an era of rapidly emerging and effective pharmacological therapies for advanced $\mathrm{PCa}$, for which ADT remains a mainstay of treatment.

Competing interests: Dr. So has been an advisory board member for AbbVie, Amgen, Astellas, Bayer, Janssen, Ferring, and TerSera; and has participated in clinical trials supported by Astellas, Ferring, and Janssen. Dr. Izard has received a grant(s) or honoraria from AbbVie, Astellas, Bayer, Ferring, Janssen, and Sanofi; and has participated in clinical trials supported by AbbVie, Astellas, AstraZeneca, Bayer, Janssen, and Merck. Dr. Saad has been an advisory board member for and has received payment/honoraria from AbbVie, Amgen, Astellas, Bayer, Janssen, and Sanofi; and has participated in clinical trials supported by Amgen, Astellas, Bayer, Janssen, and Sanofi. Dr. Shayegan has been an advisory board member for Astellas, Bayer, and Janssen; and has received a research grant from Janssen. Dr. Aprikian has been an advisory board member for AbbVie, Astellas, and Bayer; and has received grants from AbbVie, Astellas Bayer, Sanofi, and TerSera. Dr. Rendon has been an advisory board and speakers' bureau member for and has received honoraria from AbbVie, Amgen, Astellas, AstraZeneca, Bayer, Ferring, Jansen, and Sanofi. The remaining authors report no competing personal or financial interests related to this work..

Prior to publication, the full-text version of this guideline was reviewed and approved by the CUA Guidelines Committee, CUA members at large, and the CUA Board.

Correspondence: Dr. Ricardo Rendon, Department of Urology, Dalhousie, University, Halifax, NS, Canada; rrendon@dal.ca 